\title{
Stripe-hexagon competition in forced pattern forming systems with broken up-down symmetry
}

\author{
R. Peter, M. Hilt, F. Ziebert, J. Bammert, C. Erlenkämper, N. \\ Lorscheid, C. Weitenberg, A. Winter, M. Hammele, and W. Zimmermann \\ Theoretische Physik, Universität des Saarlandes, D-66041 Saarbrücken, Germany
}

(Dated: October 21, 2004)

\begin{abstract}
We investigate the response of two-dimensional pattern forming systems with a broken up-down symmetry, such as chemical reactions, to spatially resonant forcing and propose related experiments. The nonlinear behavior immediately above threshold is analyzed in terms of amplitude equations suggested for a $1: 2$ and 1:1 ratio between the wavelength of the spatial periodic forcing and the wavelength of the pattern of the respective system. Both sets of coupled amplitude equations are derived by a perturbative method from the Lengyel-Epstein model describing a chemical reaction showing Turing patterns, which gives us the opportunity to relate the generic response scenarios to a specific pattern forming system. The nonlinear competition between stripe patterns and distorted hexagons is explored and their range of existence, stability and coexistence is determined. Whereas without modulations hexagonal patterns are always preferred near onset of pattern formation, single mode solutions (stripes) are favored close to threshold for modulation amplitudes beyond some critical value. Hence distorted hexagons only occur in a finite range of the control parameter and their interval of existence shrinks to zero with increasing values of the modulation amplitude. Furthermore depending on the modulation amplitude the transition between stripes and distorted hexagons is either sub- or supercritical.
\end{abstract}

PACS numbers: $82.40 . \mathrm{Ck}, 47.20 . \mathrm{Ky}, 47.54 .+\mathrm{r}$

\section{INTRODUCTION}

External periodic forcing provides a powerful tool to analyze the response behavior of nonlinear pattern forming systems, allowing for instance the study of their inherently nonlinear mechanism of self organization. The early investigations of effects dealing with spatially periodic forcing were devoted to pattern formation in hydrodynamic systems followed by chemical systems $1,2,2,3,4,5$, 6, 17, 8, 9, 10, 11, 12, 13, 14, 15, 16, 17, 18, 19, 20, 21, 22]. The situation is comparable for temporal forcing of patterns $23,24,25,26,27,28,29,30,31,32]$ whereas recent investigations on spatiotemporal forcing have mainly been motivated by chemical reactions [33, 34, 35, 36].

In thermal convection, when deviations from the Boussinesq approximation come into play [37, 38], as well as in several chemical reactions of the Turing type [39, 40], the up-down symmetry of the fields describing the patterns, $u(\mathbf{r}, t) \rightarrow-u(\mathbf{r}, t)$, is broken. In such twodimensional extended systems rotationally symmetric in a plane, hexagons are the generic pattern close to threshold. In systems with a strong axial anisotropy, such as in nematic liquid crystals, this rotational symmetry is broken 41, 42, 43, 44] and hexagonal convection patterns do not occur. Thus the interplay between different broken symmetries, such as the broken up-down symmetry with a weak anisotropy, leads to an interesting competition between stripe and hexagonal patterns as has been shown recently [45, 46, 47].

Which kind of effects may be expected if besides the up-down symmetry the translational symmetry is simultaneously broken by periodic modulation in one spatial direction? As the direction of the modulation wave- number defines a preferred direction the system becomes anisotropic. In the following we present the symmetry adapted amplitude equations and analyze the related hexagon-stripe competition in terms of these equations. In thermal convection this symmetry breaking may be achieved by modulating the container boundaries or the applied temperature difference periodically in one direction 1, 8, 14]. In chemical reactions however, the forcing is introduced by a spatially modulated illumination of the system [20, 48]. The specific manifestation of the modulation-induced symmetry breaking in generic amplitude equations depends on the ratio between the modulation wave number $k_{m}$ and the wave number $k_{c}$ of the respective pattern. We will study two cases of resonant modulation: $k_{m}=2 k_{c}$ and $k_{m}=k_{c}$.

Exemplarily we will focus in this work on the LengyelEpstein model for a chemical reaction. This model, introduced in Sec. III displays in some parameter range a Turing instability of the homogeneous basic state as will be shown in Sec. IIII Immediately above the threshold of a supercritical bifurcation the amplitudes of the unstable pattern forming modes are still small enough to apply the powerful perturbational technique of amplitude equations. In Sec.IV we derive the amplitude equations from the given Lengyel-Epstein model in the limit of small spatially periodic modulations of the illumination acting on the chemical reactions. We consider a resonant ratio of $1: 1$ as well as of $1: 2$ between the wavenumber of the Turing instability and the modulation wavenumber. The amplitude equations obtained in each of these resonant cases are generic and may be found for other systems having the same symmetry properties as the considered Lengyel-Epstein model, for instance modulated 
convection systems. Without spatially periodic forcing it is known that the resulting amplitude equations favor hexagonal patterns close to threshold. In Sec. V we investigate how the spatial forcing modifies the transition scenario between stripe and hexagon-like patterns and how the initial hexagonal patterns get distorted. Analytical calculations are confirmed by numerical simulations in Sec. VI Concluding remarks and an outlook on traveling stripe forcing within the scope of the underlying chemical reaction are given in Sec. VII

\section{THE LENGYEL-EPSTEIN MODEL}

As a basic model of a chemical reaction we choose the Lengyel-Epstein model as described in Ref. 49]. If the effect of illumination on the chemical reaction is taken into account, as done in Ref. [50], the model may be described by the following equations

$$
\begin{aligned}
& \partial_{t} u=a-c u-4 \frac{u v}{1+u^{2}}-\Phi+\Delta u \\
& \partial_{t} v=\sigma\left(c u-\frac{u v}{1+u^{2}}+\Phi+d \Delta v\right) .
\end{aligned}
$$

In these equations the dimensionless concentrations of two relevant chemical substances are labelled with $u(\mathbf{r}, t)$ and $v(\mathbf{r}, t)$, whereas $a, c, \sigma$ and $d$ denote dimensionless parameters of the chemical system. Finally the effect of external illumination is described by the field $\Phi(\mathbf{r}, t)$, which can be identified as the control parameter of the system and decomposed into a spatially homogeneous and a spatially periodic part [20, 21, 51]

$$
\Phi(\mathbf{r}, t)=\phi+M(\mathbf{r})
$$

For the second term of Eq. (2) we assume a periodic dependence in one spatial direction as described by

$$
M(\mathbf{r})=2 \tilde{G} \cos \left(k_{m} x\right) .
$$

For a spatially homogeneous illumination Eqs. (11) have a stationary and homogeneous solution given by

$$
u_{0}=\frac{a-5 \phi}{5 c}, \quad v_{0}=\frac{a\left(1+u_{0}^{2}\right)}{5 u_{0}} .
$$

\section{THRESHOLD FOR PATTERN FORMATION}

In a certain parameter range the spatially homogeneous state described by Eqs. (4) becomes unstable against infinitesimal perturbations $u_{1}(\mathbf{r}, t)$ and $v_{1}(\mathbf{r}, t)$. In order to determine this interval the basic state is separated from the inhomogeneous contributions to both fields by the ansatz

$$
\begin{aligned}
& u(\mathbf{r}, t)=u_{0}+u_{1}(\mathbf{r}, t) \\
& v(\mathbf{r}, t)=v_{0}+v_{1}(\mathbf{r}, t) .
\end{aligned}
$$

In a next step the linerization of the basic Eqs. (1) with respect to those small contributions $u_{1}(\mathbf{r}, t)$ and $v_{1}(\mathbf{r}, t)$ yields linear equations with constant coefficients, which may be solved by the mode solutions

$$
u_{1}(\mathbf{r}, t)=\bar{u}_{1} e^{\lambda t+i \mathbf{k} \cdot \mathbf{r}}, \quad v_{1}(\mathbf{r}, t)=\bar{v}_{1} e^{\lambda t+i \mathbf{k} \cdot \mathbf{r}}
$$

and thus leads to a set of homogeneous linear equations for the amplitudes $\bar{u}_{1}$ and $\bar{v}_{1}$. From these equations the dispersion relation $\lambda(k)$ may be specified. It turns out that a strong and homogeneous illumination of the chemical reaction suppresses inhomogeneous perturbations. This is illustrated by Fig. 1] wherein the dispersion relation $\lambda(k)$ is plotted for three different cases of the illumination strength $\phi$.

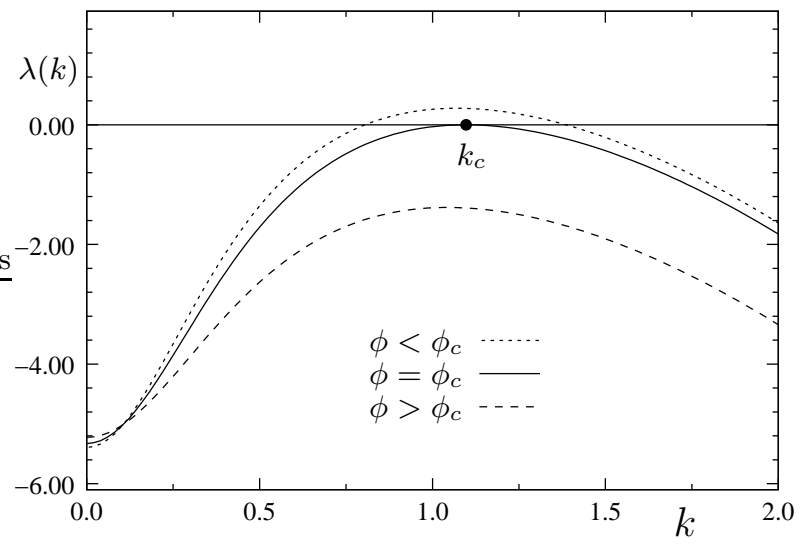

FIG. 1: The dispersion relation $\lambda(k)$ for a supercritical value $\phi<\phi_{c}$ (dotted line) of the illumination strength $\phi$, for a critical value $\phi=\phi_{c}$ (solid line) and for a subcritical value $\phi>\phi_{c}$ (dashed line). Parameters are $a=16, c=1.0, d=$ $1.5, \sigma=301$.

From the neutral stability condition

$$
\operatorname{Re}(\lambda(k))=0, \quad \Rightarrow \phi_{0}(k)
$$

the neutral curve $\phi_{0}(k)$ may be determined, which has to be done numerically in our case. Some examples of characteristic neutral curves are plotted in Fig. 2 for different values of the diffusion coefficient $d$. For $d=1.2$ the homogeneous state is either stable for a small or for a strong illumination $\phi$ and is thus unstable only within a small island enclosed by the corresponding neutral curve in the $\phi$ - $k$-plane. However, for $d>1.4$ the homogeneous state remains only stable for strong illuminations. In this case the neutral curve $\phi_{0}(k)$ takes its maximum at the wavenumber

$$
k_{c}^{2}=\frac{1}{2}\left(\frac{4 v_{0}\left(u_{0}^{2}-1\right)}{\left(1+u_{0}^{2}\right)^{2}}-c-\frac{u_{0}}{d\left(1+u_{0}^{2}\right)}\right)
$$

and the value of the illumination at the maximum of the neutral curve defines the critical illumination strength $\phi_{c}=\phi_{o}\left(k_{c}\right)$. This critical value is plotted in Fig. 3] as a 


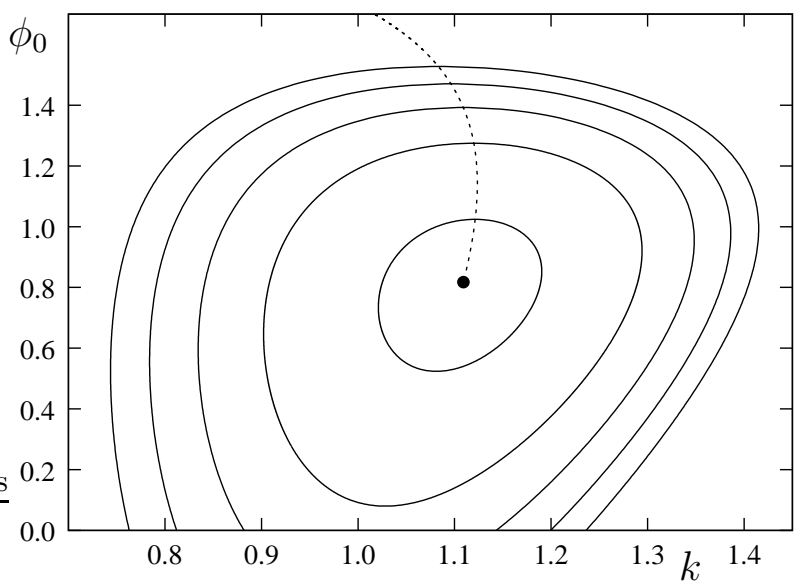

FIG. 2: The neutral curve $\phi_{0}(k)$ is determined numerically via the neutral stability condition in Eq. (7) for different values of the diffusion parameter $d=1.2,1.3, \ldots, 1.6$ (from inside to outside). The remaining parameters are $a=16, c=1.0, \sigma=$ 301 in all cases. The homogeneous state is unstable inside of each curve. The dotted line shows $k_{c}$ as a function of $d$.

function of the diffusion coefficient $d$ and for various values of the parameter $c$. The occurring Turing instability vanishes, when the inner closed curve in Fig. 2] shrinks to zero, i.e. below $d_{c}=1.179$ for the parameter set chosen in this figure. Below this critical value $d_{c}$ the stationary homogeneous state becomes stable with respect to an oscillatory instability.

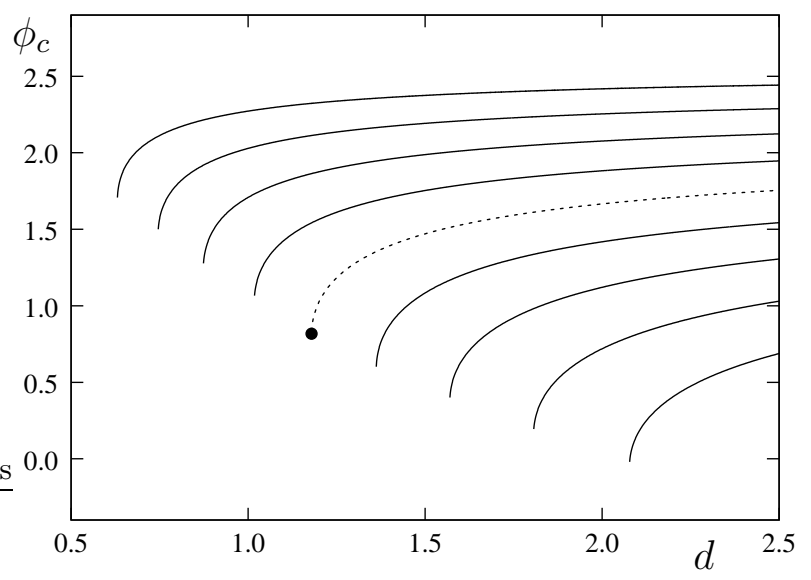

FIG. 3: The critical illumination $\phi_{c}=\phi_{0}\left(k_{c}\right)$ is plotted as a function of the diffusion coefficient $d$ for different values of the parameter $c$, with $c=0.6,0.7,0.8, \ldots, 1.4$ from top to bottom and $a=16, \sigma=301$. The dotted line corresponds to that in Fig. 2

\section{AMPLITUDE EQUATIONS AND LINEAR STABILITY OF THEIR SOLUTIONS}

The amplitudes $\bar{u}_{1}$ and $\bar{v}_{1}$ of the linear modes described by Eq. (6) as well as the amplitudes of any other pattern are restricted by the nonlinear terms proportional to $u_{1}$ and $v_{1}$ in Eqs. (11). In two-dimensional systems with a broken up-down symmetry the common patterns close to threshold are stripes, squares or hexagons 52]. Immediately beyond a supercritical bifurcation, where the amplitude of an emerging pattern is still small, the concept of the so-called amplitude equations is a very successful one to characterize the nonlinear behavior of patterns, as it is exemplified for several physical, chemical and biological systems in Refs. [52, 53. . The amplitude equations are obtained by a perturbative method from the basic Eqs. (11), whereby the coefficients occurring in these amplitude equations reflect the dependence of the patterns on the parameters of the specific system.

\section{A. Scheme of the derivation of amplitude equations}

The small parameter used in the perturbative derivation of the amplitude equations is the relative distance to the threshold $\phi_{c}$

$$
\varepsilon=\frac{\phi_{c}-\phi}{\phi_{c}}
$$

Since the up-down symmetry for inhomogeneous field contributions is broken, i.e. $u_{1}, v_{1} \nrightarrow \nrightarrow-u_{1},-v_{1}$, we expect hexagonal patterns to occur in some parameter ranges. Close to threshold hexagonal patterns are described by a superposition of three waves (stripe patterns) with wave vectors enclosing an angle of about $120^{\circ}$ with respect to each other. If the two fields $u_{1}$ and $v_{1}$ are rewritten in terms of a vector $\mathbf{w}_{1}=\left(u_{1}, v_{1}\right)^{T}$, the generating field of hexagonal patterns may be represented by

$$
\mathbf{w}_{1}=\left(\begin{array}{c}
1 \\
E_{0}
\end{array}\right)\left(A_{1} e^{i \mathbf{k}_{1} \mathbf{r}}+A_{2} e^{i \mathbf{k}_{2} \mathbf{r}}+A_{3} e^{i \mathbf{k}_{3} \mathbf{r}}\right)+c . c .,
$$

where the wave vectors $\mathbf{k}_{i}(i=1,2,3)$ of the three underlying stripe patterns read

$$
\mathbf{k}_{1}=k_{c}\left(\begin{array}{l}
1 \\
0
\end{array}\right) \quad \text { and } \quad \mathbf{k}_{2,3}=\frac{k_{c}}{2}\left(\begin{array}{c}
-1 \\
\pm \sqrt{3}
\end{array}\right)
$$

and the second component $E_{0}$ of the eigenvector is given by

$$
E_{0}=-\left(c+k_{c}^{2}\right) \frac{1+u_{0}^{2}}{4 u_{0}}-\frac{v_{0}}{u_{0}} \frac{1-u_{0}^{2}}{1+u_{0}^{2}} .
$$

The coupled amplitude equations deduced for the three envelope functions $A_{i}(i=1,2,3)$ are identical apart from 
the term evoked by the forcing which naturally differs whether we choose in Eq. (3) a ratio $1: 2=k_{c}: k_{m}$ between the modulation wave number $k_{c}$ and the critical wave number $k_{m}$ or a ratio $1: 1=k_{c}: k_{m}$. In the first case the modulation amplitude $G$ should vary as $G=G_{1} \propto \varepsilon$, in the latter one as $G=G_{2} \propto \varepsilon^{3 / 2}$.

Choosing the ansatz specified in Eq. (10) and expanding Eq. (1) with respect to powers of the small parameter $\varepsilon$ as well as the small amplitudes $A_{i}$ one obtains the following set of nonlinear equations for the three amplitudes $A_{i}$

$$
\begin{aligned}
\tau_{0} \partial_{t} A_{1}=\varepsilon & A_{1}+\delta A_{2}^{*} A_{3}^{*}+G_{1} A_{1}^{*}+G_{2} \\
& -\left(\gamma\left|A_{1}\right|^{2}+\rho\left|A_{2}\right|^{2}+\rho\left|A_{3}\right|^{2}\right) A_{1}, \\
\tau_{0} \partial_{t} A_{2}= & \varepsilon A_{2}+\delta A_{3}^{*} A_{1}^{*} \\
& -\left(\gamma\left|A_{2}\right|^{2}+\rho\left|A_{3}\right|^{2}+\rho\left|A_{1}\right|^{2}\right) A_{2}, \\
\tau_{0} \partial_{t} A_{3}=\varepsilon & A_{3}+\delta A_{1}^{*} A_{2}^{*} \\
& -\left(\gamma\left|A_{3}\right|^{2}+\rho\left|A_{1}\right|^{2}+\rho\left|A_{2}\right|^{2}\right) A_{3},
\end{aligned}
$$

which are quite similar to those presented in Refs. 35, 45, 54]. Depending on which of the resonant cases mentioned above is considered either $G_{1}$ or $G_{2}$ is nonzero. For further details on the derivation scheme of the amplitude equations we refer to Ref. [55], where likewise reaction diffusion equations were studied in detail.

The analytical expressions of the coefficients $\tau_{0}, \delta, \gamma, \rho$ appearing in the amplitude equations (13) as functions of the parameters of the basic equations (II) are rather lengthy. Instead of giving their analytical forms we therefore plot them in Fig. 4 as functions of the diffusion coefficient $d$ while assuming different values for $c$. Rescaling
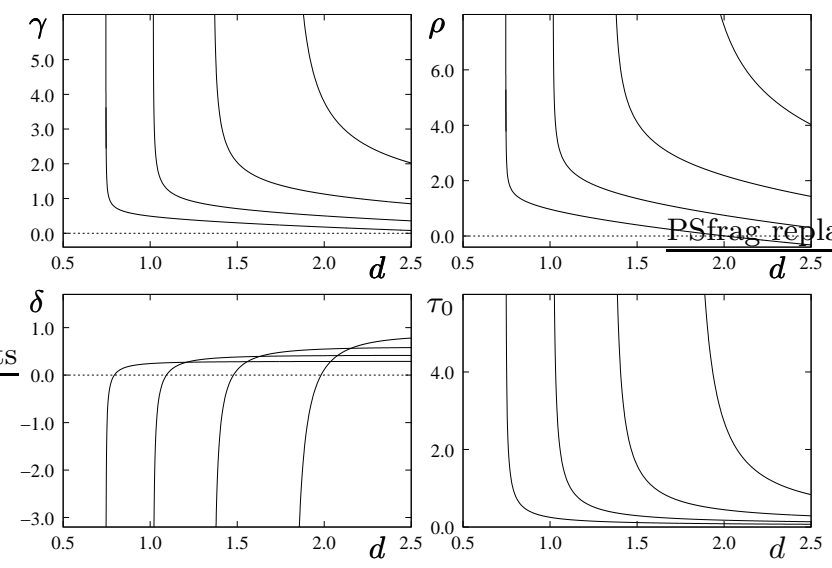

FIG. 4: The coefficients of the amplitude equations in Eq. (13) are shown close to the $1: 2$ resonance, i.e. $G_{2}=0$, as functions of the diffusion coefficient $d$ for different values of the parameter $c$, from left to right $c=0.7,0.9,1.1,1.3$.

of the amplitudes $A_{i}(i=1,2,3)$ allows to express time as well as the coefficients of Eqs. (13) as follows

$$
\begin{aligned}
& \bar{A}=\frac{\gamma}{|\delta|} A, \quad \eta=\frac{\gamma}{\delta^{2}} \varepsilon, \quad \bar{t}=\frac{\delta^{2}}{\tau_{0} \gamma} t, \\
& \bar{G}_{1}=\frac{\gamma}{\delta^{2}} G_{1}, \quad \bar{G}_{2}=\frac{\gamma^{2}}{|\delta|^{3}} G_{2}, \\
& \bar{\rho}=\frac{\rho}{\gamma}, \quad \bar{\delta}=\frac{\delta}{|\delta|}
\end{aligned}
$$

and yields a simpler form of Eqs. (13)

$$
\begin{gathered}
\partial_{\bar{t}} \bar{A}_{1}=\eta \bar{A}_{1}+\bar{\delta} \bar{A}_{2}^{*} \bar{A}_{3}^{*}+\bar{G}_{1} \bar{A}_{1}^{*}+\bar{G}_{2} \\
-\left(\left|\bar{A}_{1}\right|^{2}+\bar{\rho}\left|\bar{A}_{2}\right|^{2}+\bar{\rho}\left|\bar{A}_{3}\right|^{2}\right) \bar{A}_{1}, \\
\partial_{\bar{t}} \bar{A}_{2}=\eta \bar{A}_{2}+\bar{\delta} \bar{A}_{3}^{*} \bar{A}_{1}^{*} \\
\quad-\left(\left|\bar{A}_{2}\right|^{2}+\bar{\rho}\left|\bar{A}_{3}\right|^{2}+\bar{\rho}\left|\bar{A}_{1}\right|^{2}\right) \bar{A}_{2}, \\
\partial_{\bar{t}} \bar{A}_{3}=\eta \bar{A}_{3}+\bar{\delta} \bar{A}_{1}^{*} \bar{A}_{2}^{*} \\
-\left(\left|\bar{A}_{3}\right|^{2}+\bar{\rho}\left|\bar{A}_{1}\right|^{2}+\bar{\rho}\left|\bar{A}_{2}\right|^{2}\right) \bar{A}_{3}
\end{gathered}
$$

with $\bar{\delta}= \pm 1$. Choosing $\tau_{0}=\gamma=1$ and $\delta= \pm 1$ in Eqs. (13) reduces them to the same form as Eqs. (15) which besides the modulation amplitude $G_{i}(i=1,2)$ and the control parameter $\eta$ only depend on the nonlinear coefficient $\bar{\rho}$. In most cases this coefficient takes values in the interval $\bar{\rho} \in[1,2]$ as shown in Fig. [5 where $\bar{\rho}$ is plotted as a function of the diffusion coefficient $d$ for different values of $c$.

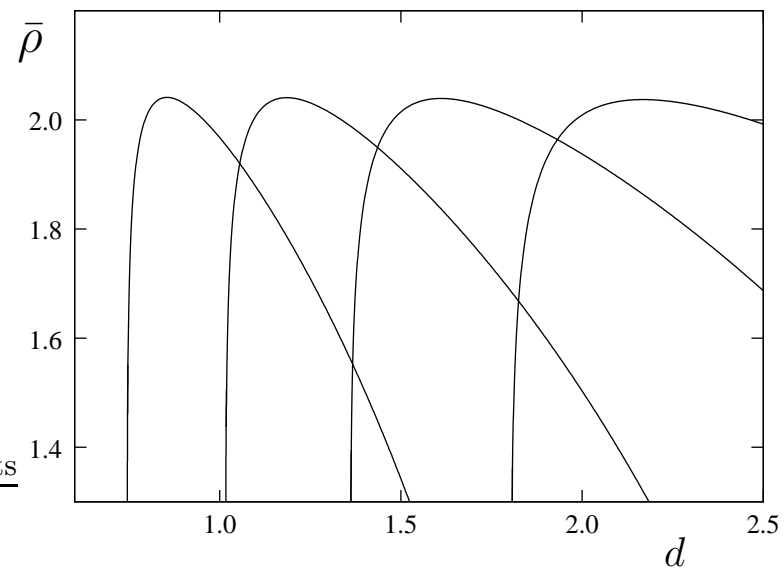

FIG. 5: The rescaled coefficient $\bar{\rho}=\rho / \gamma$ as a function of $d$ for various values of $c$, with $c=0.7,0.9,1.1,1.3$ from left to right.

\section{B. Functional}

The coupled nonlinear equations (13) may be considered as a potential dynamics

$$
\tau_{0} \partial_{t} A_{i}=-\frac{\delta \mathcal{F}}{\delta A_{i}^{*}}
$$


with the functional

$$
\begin{aligned}
\mathcal{F}=\sum_{i=1}^{3} & \left(\frac{\gamma}{2}\left|A_{i}\right|^{4}-\varepsilon\left|A_{i}\right|^{2}\right)+\frac{\rho}{2} \sum_{j \neq i}^{3}\left|A_{i}\right|^{2}\left|A_{j}\right|^{2} \\
& -\frac{G_{1}}{2}\left(A_{1}^{2}+A_{1}^{* 2}\right)-G_{2}\left(A_{1}+A_{1}^{*}\right) \\
& -\delta\left(A_{1} A_{2} A_{3}+A_{1}^{*} A_{2}^{*} A_{3}^{*}\right) .
\end{aligned}
$$

This functional or its rescaled version yielding Eqs. 15 provides a useful criterion to decide which kind of pattern is most likely to occur in a given parameter range. Generally the pattern leading to the lowest value of the functional is preferred. Once again either $G_{1}$ or $G_{2}$ is nonzero, corresponding to one of the considered resonant cases.

\section{Linear stability of hexagons and rolls}

Since we only consider a spatially periodic modulation in $x$-direction, the translational invariance in $y$-direction is preserved. This holds for both resonant cases under investigation. Therefore two out of three amplitudes, namely $A_{2}$ and $A_{3}$ are degenerated and the remaining two stationary amplitudes, called $A$ and $B$, are determined by two of the three Eqs. (15). The linear stability of the stationary and nonlinear solutions is analyzed by superimposing small perturbations to the latter ones, meaning we use the following ansatz:

$$
\bar{A}_{1}=A+\mu_{1}(\bar{t}), \quad \bar{A}_{2,3}=B+\mu_{2,3}(\bar{t}) .
$$

Accordingly the amplitude equations (15) are linearized with respect to the small perturbations $\mu_{i}(i=1,2,3)$ which leads to the following set of equations

$$
\begin{aligned}
\partial_{\bar{t}} \mu_{1}= & \left(\eta-2|A|^{2}-2 \bar{\rho}|B|^{2}\right) \mu_{1}+\left(\bar{G}_{1}-A^{2}\right) \mu_{1}^{*} \\
- & \bar{\rho} A B^{*}\left(\mu_{2}+\mu_{3}\right)+\left(\bar{\delta} B^{*}-\bar{\rho} A B\right)\left(\mu_{2}^{*}+\mu_{3}^{*}\right) \\
\partial_{\bar{t}} \mu_{2}= & \left(\eta-(2+\bar{\rho})|B|^{2}-\bar{\rho}|A|^{2}\right) \mu_{2}-B^{2} \mu_{2}^{*} \\
& -\bar{\rho} A^{*} B \mu_{1}+\left(\bar{\delta} B^{*}-\bar{\rho} A B\right) \mu_{1}^{*} \\
& -\bar{\rho}|B|^{2} \mu_{3}+\left(\bar{\delta} A^{*}-\bar{\rho} B^{2}\right) \mu_{3}^{*} \\
\partial_{\bar{t}} \mu_{3}= & \left(\eta-(2+\bar{\rho})|B|^{2}-\bar{\rho}|A|^{2}\right) \mu_{3}-B^{2} \mu_{3}^{*} \\
& -\bar{\rho} A^{*} B \mu_{1}+\left(\bar{\delta} B^{*}-\bar{\rho} A B\right) \mu_{1}^{*} \\
& -\bar{\rho}|B|^{2} \mu_{2}+\left(\bar{\delta} A^{*}-\bar{\rho} B^{2}\right) \mu_{2}^{*} .
\end{aligned}
$$

These coupled equations may be transformed into a set of homogeneous linear equations by means of an ansatz for the perturbations

$$
\mu_{i}=\nu_{1 i} e^{\sigma t}+\nu_{2 i}^{*} e^{\sigma^{*} t} \quad(i=1,2,3)
$$

which allows to determine the growth rate $\sigma(k, \ldots)$ of those perturbations. Marginal stability, i.e. $\operatorname{Re}[\sigma(k, \ldots)]=0$, then yields the stability boundary as a function of the control parameter $\eta$. As an example, if one considers the stability of stripes in a $2: 1$ resonant case, the amplitude $B=0$ and the marginal stability condition leads to a polynomial in $\eta$

$$
\begin{aligned}
(\bar{\rho}-1) \eta^{2} & +\left[2 \bar{\rho} \bar{G}_{1}(\bar{\rho}-1)-\bar{\delta}^{2}\right] \eta \\
& -\bar{G}_{1}\left(\bar{\delta}^{2}-\bar{\rho}^{2} \bar{G}_{1}\right)=0,
\end{aligned}
$$

whose solutions are

$$
\begin{aligned}
\eta_{1,2}= & \frac{2 \bar{\rho} \bar{G}_{1}(1-\bar{\rho})+\bar{\delta}^{2}}{2(1-\bar{\rho})^{2}} \\
& \pm \frac{\bar{\delta}}{2(\bar{\gamma}-\bar{\rho})^{2}} \sqrt{\bar{\delta}^{2}+4 \bar{G}_{1}(1-\bar{\rho})} .
\end{aligned}
$$

Consequently single mode solutions (stripes) are linear unstable within the region bounded by those values of $\eta$ and linear stable in the complementary area.

\section{NONLINEAR SOLUTIONS OF THE MODULATED AMPLITUDE EQUATIONS}

In this section the bifurcation-scenarios from the homogeneous basic state and thus the weakly nonlinear solutions of Eqs. (15) are discussed for three different cases: first of all we consider an unmodulated system $\left(G_{1}=G_{2}=0\right)$, then we study the modulated cases already taken into account in Sec.IV namely either $G_{1} \neq 0$ and $G_{2}=0$ or $G_{1}=0$ and $G_{2} \neq 0$. Without modulations one has either a single mode solution with modulus $\left|\bar{A}_{1}\right|=A$ and $\left|\bar{A}_{2}\right|=\left|\bar{A}_{3}\right|=0$, or hexagons corresponding to a three mode solution with coinciding moduli $\left|\bar{A}_{1}\right|=\left|\bar{A}_{2}\right|=\left|\bar{A}_{3}\right|$. In case of a finite modulation amplitude single mode solutions as well as three mode solutions with amplitudes satisfying $\left|\bar{A}_{1}\right| \neq\left|\bar{A}_{2}\right|=\left|\bar{A}_{3}\right|$ may be encountered. By means of the determined nonlinear solutions the functional value of the latter ones may be calculated through Eq. (17), which gives rise to the intervals wherein either the one mode or the three mode solution is preferred.

\section{A. Solutions of the unforced amplitude equations}

Without modulations Eqs. (15) have either a single mode solution $|\bar{A}|=\sqrt{\eta}$ or a three mode solution with equal moduli, i.e. with amplitudes fulfilling $\left|\bar{A}_{1}\right|=$ $\left|\bar{A}_{2}\right|=\left|\bar{A}_{3}\right|=A$. Assuming a relative phase shift $\theta_{j}$ for each linear mode contributing to the three mode solution

$$
\bar{A}_{j}=A e^{i \Theta_{j}}
$$

one obtains a nonlinear equation for the common modulus $A$

$$
0=\eta A+\bar{\delta} e^{i \Theta} A^{2}-(1+2 \bar{\rho}) A^{3},
$$

with $\bar{\delta}= \pm 1$ and the sum of the phase angles $\Theta=\theta_{1}+$ $\theta_{2}+\theta_{3}$. Eq. (24) has two real solutions

$$
A_{ \pm}=\frac{1}{2(1+2 \bar{\rho})}\left[\bar{\delta} \pm \sqrt{\bar{\delta}^{2}+4 \eta(1+2 \bar{\rho})}\right]
$$


where $A_{+}$corresponds to the larger one of the two amplitudes with $\bar{\delta}=1$, while $A_{-}$is obtained for $\bar{\delta}=-1$. If $\bar{\delta}=1$ the phase angle is $\Theta=0$ and one expects regular hexagons whereas $\bar{\delta}=-1$ yields a phase angle $\Theta=\pi$ corresponding to inverse hexagons. Comparing the functional given in Eq. (17) of those solutions one may ascertain: In case $\bar{\delta}=1$ regular hexagons are preferred as $\mathcal{F}_{H}^{+}<\mathcal{F}_{H}^{-}$and if $\bar{\delta}=-1$ inverse hexagons are most likely to occur because their functional value is the lowest one, i.e. $\mathcal{F}_{H}^{-}<\mathcal{F}_{H}^{+}$.

\section{B. Solutions of the forced amplitude equations for $k_{m}=2 k_{c}$ and $G_{1} \neq 0, G_{2}=0$.}

In the case of a $2: 1$ resonance with a finite modulation amplitude $G_{1}$ two of the three amplitudes coincide $A_{2,3}=B e^{i \theta_{2,3}}$, the third one being $A_{1}=A e^{i \theta_{1}}$. Thus the remaining two amplitudes $A$ and $B$ are determined by the following two nonlinear equations

$$
\begin{aligned}
& \left(\eta-A^{2}-2 \bar{\rho} B^{2}\right) A+\bar{G}_{1} A+\bar{\delta} e^{i \Theta} B^{2}=0, \\
& \left(\eta-(1+\bar{\rho}) B^{2}-\bar{\rho} A^{2}\right) B+\bar{\delta} e^{i \Theta} A B=0,
\end{aligned}
$$

with $\Theta=\theta_{1}+\theta_{2}+\theta_{3}$. The single mode solution generated by those coupled equations is given by

$$
A=\sqrt{\eta+\bar{G}_{1}}, \quad B=0
$$

and bifurcates supercritcally at the threshold $\varepsilon=-G_{1}$ or $\eta=-\bar{G}_{1}$ in rescaled units. A linear stability analysis of the single mode solution according to the scheme described in Sec. IVC shows that the single mode is linear unstable in the region delimited by the two values of $\eta$

$$
\begin{aligned}
\eta_{1,2}= & \frac{2 \bar{\rho} \bar{G}_{1}(1-\bar{\rho})+\bar{\delta}^{2}}{2(1-\bar{\rho})^{2}} \\
& \pm \frac{\bar{\delta}}{2(\bar{\gamma}-\bar{\rho})^{2}} \sqrt{\bar{\delta}^{2}+4 \bar{G}_{1}(1-\bar{\rho})} .
\end{aligned}
$$

The three mode solution with $\bar{\delta} e^{i \Theta}=1$ is generally preferred and the appendant amplitudes $A$ and $B$ may be determined by solving Eqs. (26) with a standard computer algebra program. Instead of presenting the rather complex analytical solutions we opt for the characteristic bifurcation diagrams based on four different parameter sets in Fig. [6] which ought to cover the typical bifurcation scenarios encountered. In Fig. 6 the linear stable intervals of each branch are visualized by solid lines, whereas unstable ones are represented by dashed lines.

Part (a) of Fig. 6] shows the well known bifurcation scenario of the unmodulated case $\left(\bar{G}_{1}=0\right)$ for supercritcally bifurcating stripes and subcritically bifurcating hexagonal solutions [54]. With increasing values of the modulation amplitude $\bar{G}_{1}$ the degeneracy of the three mode solution vanishes and only the onset of the single mode solution starts at $\eta_{c}=-\bar{G}_{1}$. This removal of degeneracy is already best reflected in Fig. 6 (b), wherein $\bar{G}_{1}=0.05$ :
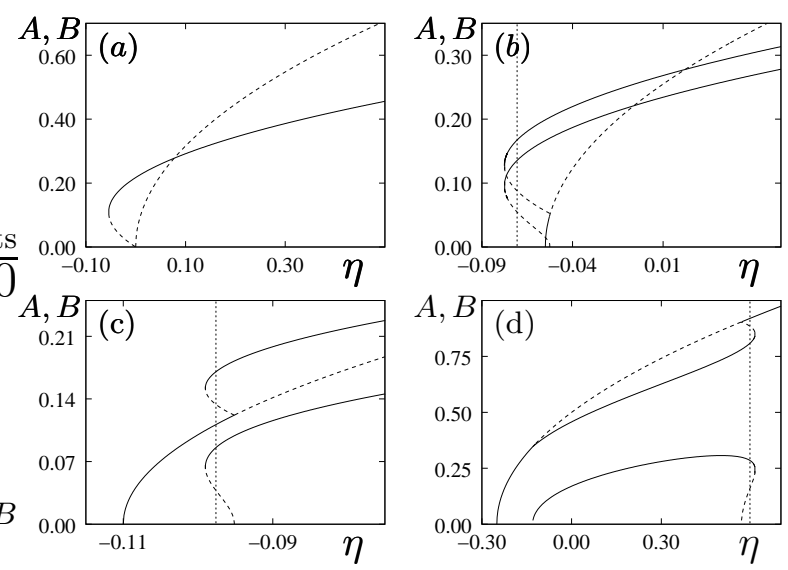

FIG. 6: The amplitude of supercritically bifurcating stripes and the subcritically bifurcating hexagons with three degenerated amplitudes are shown in (a) as a function of $\eta$ for the unmodulated case. In each case the solid lines mark the linear stable part of each branch and the dashed parts the unstable ones. For finite modulation amplitudes $\bar{G}_{1}$ the three mode solution with $A$ always corresponding to the upper branch and $B$ to the lower branch, as well as the amplitude of a single mode solution are plotted as a function of the control parameter $\eta$ for three different values of the modulation amplitude $G_{1}=0.05$ (b), $G_{1}=0.11$ (c) and $G_{1}=0.25$ (d) whereas the nonlinear coefficient $\bar{\rho}$ is fixed at $\bar{\rho}=1.8$ and $\bar{\delta}=1$. Each vertical dotted line reflects the $\eta$-value where the values of the functional $\mathcal{F}$ of the one and the three mode solution coincide.

the subcritical branches of the hexagonal solution, $A$ corresponding to the larger amplitude and $B$ to the smaller one, do not overlap anymore. For finite values of $\bar{G}_{1}$ the upper hexagon-branch, i.e. the $A$-branch, always emerges from the single mode solution, while the $B$-branch which is the lower hexagon-branch bifurcates from zero with decreasing values $\eta$. If $\bar{G}_{1}$ is small enough the bifurcation of the three-mode solution is subcritical as depicted in Fig. [6 (b) and Fig. [6] (c), whereas for large modulation amplitudes this bifurcation is shifted to smaller $\eta$-values while becoming supercritical as sketched in Fig. 6(d).

It turns out that for intermediate values of $\bar{G}_{1}$, such as those used in Fig. 6(b) and Fig. 6(c), the three mode solution bifurcates subcritically from the single mode solution not only for small values of the control parameter $\eta$ but also for large values of $\eta$ as it may be concluded from Fig. 7 For large values of $\bar{G}_{1}$, e.g. $\bar{G}_{1}=0.25$ in Fig. 6(d), the lower bifurcation point becomes supercritical while the upper one nevertheless remains subcritical at first. By comparing the bifurcation diagrams shown in Fig. 7 and Fig. 6) (d) it can easily be seen that the existence range of the three mode solution shrinks with increasing values of the modulation amplitude. Simultaneously the hysteresis becomes less pronounced with increasing values of $\bar{G}_{1}$ changing the subcritical bifurcation to a supercritical one at small $\eta$-values as well as the upper bifurcation point from subcritical to supercrit- 

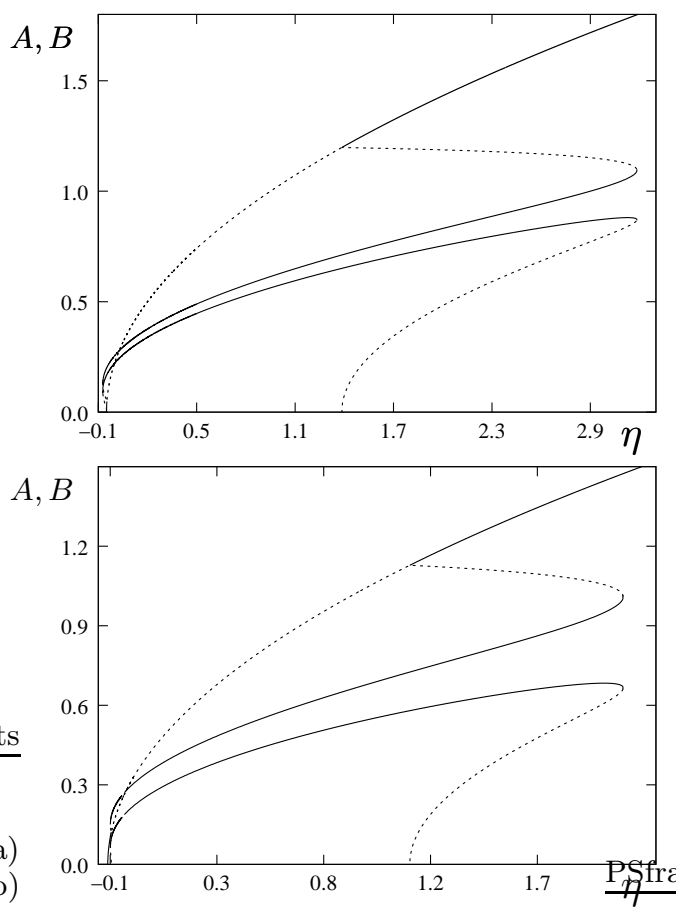

FIG. 7: The amplitudes $A(\eta)$ and $B(\eta)$ are plotted for $\bar{G}_{1}=$ 0.05 and $\bar{G}_{1}=0.11$ (already shown in Fig. 6) over a broader interval of $\eta$ which reveals that the upper bifurcation point of the three mode solution moves for increasing modulation amplitudes $\bar{G}_{1}$ to decreasing values of $\eta$. As in Fig. [ $6 \bar{\rho}=1.8$ and $\bar{\delta}=1$.

ical for even larger values of $\bar{G}_{1}$. This latter point can be nicely seen in Fig. 8 which shows that beyond some critical value $\bar{G}_{1 c}$ of the modulation amplitude the three mode solution ceases to exist. Since both bifurcations from the single to the three mode solution are already supercritical before their domain of existence shrinks to zero, the critical value $\bar{G}_{1 c}$ may be determined using the condition that the two $\eta$-values given by Eq. (28) coincide, i.e. $\eta_{1}=\eta_{2}$. Hence for modulation amplitudes $\bar{G}_{1}$ greater than the critical value

$$
\bar{G}_{1 c}=\frac{1}{4(\bar{\rho}-1)}
$$

single mode solutions are stable for all $\eta$ and three mode solutions do not exist any more. Since Fig. 5 evinces that in most cases $\bar{\rho}$ takes values larger than one, $\bar{G}_{1 c}$ should always be a positive parameter.

The domain of existence of three mode solutions, the stability range of single mode solutions as well as the points of coincidence of the corresponding functionals (vertical dotted lines) are represented in Fig. 6(b)-(d) and Fig. 7 for three different values of $\bar{G}_{1}$. These relevant properties are recapitulated as a function of the modulation amplitude $\bar{G}_{1}$ in Fig. 8 Within the region bounded by the dotted lines three mode solutions exist. Along the dashed line the functional values of one and three mode solutions match and the area lying in
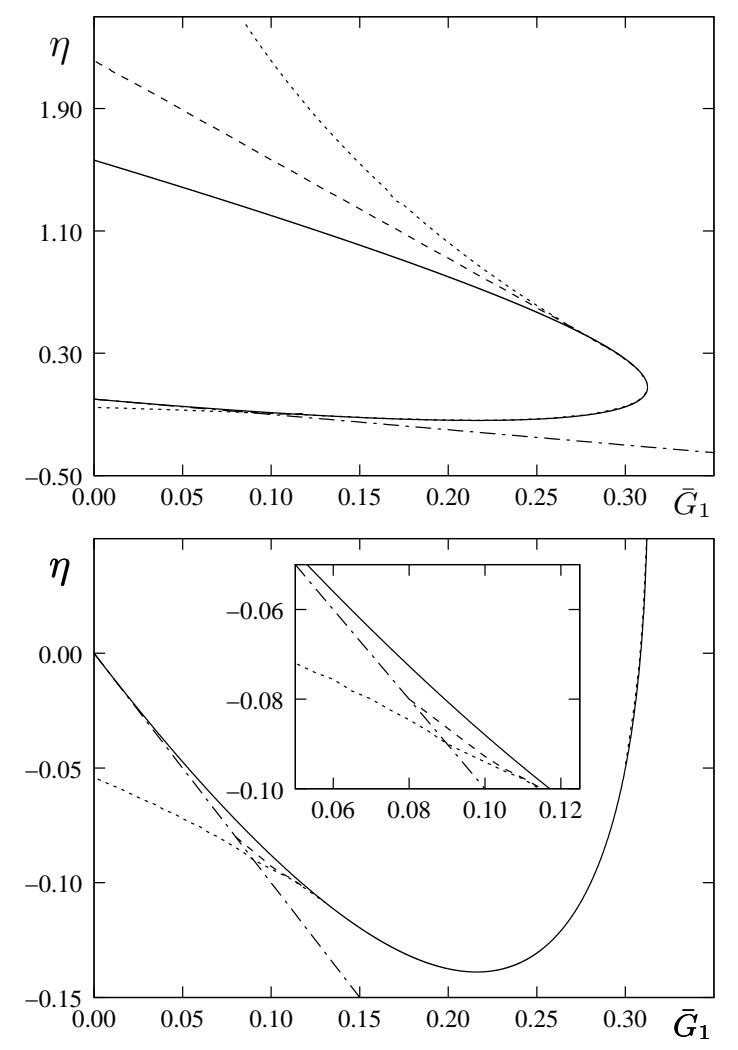

FIG. 8: Between the upper and lower dotted lines (the lower one overlapping partially with the solid line) lies the domain of existence of three mode solutions. The functionals of single and three mode solutions coincide along the dashed line (which overlaps once again partially on its lower branch with the solid one) and the region lying in between these lines is the one where three mode solutions are favored. Single mode solutions are linear unstable inside the area delimited by the solid line whereas linear stable outside and the dashed-dotted line corresponds to the threshold $\eta_{c}=-\bar{G}_{1}$ of the single mode solution. Part (b) of the figure holds an enlargement of the small $\eta$ range in order to distinguish properly the different curves in the lower half plane of part (a). Further parameters are $\bar{\rho}=1.8$ and $\bar{\delta}=1$.

between the upper and lower solid lines is the portion of the $\eta$ - $\bar{G}_{1}$-plane wherein one mode solutions are linear unstable. As predicted by Eq. (29) for $\bar{\rho}=1.8$ three mode solutions do not exist for modulation amplitudes greater than $\bar{G}_{1}=0.3125$. Before this critical value is reached the dotted and the solid lines are overlapping which means that the upper bifurcation of three mode solutions, i.e. the one occurring at large $\eta$-values, has become supercritical as it has already been mentioned above.

According to Eq. (10) different amplitudes $A$ and $B$ generate dissimilar spatial patterns as illustrated in Fig. 9 for four distinct parameter sets. Fig. 9(a) shows regular hexagons whereas in Fig. 9(b)-(d) distorted hexagons can be seen for various amplitude ratios $A / B$ corresponding to the parameters used in Fig. [6 (b) and (c). 

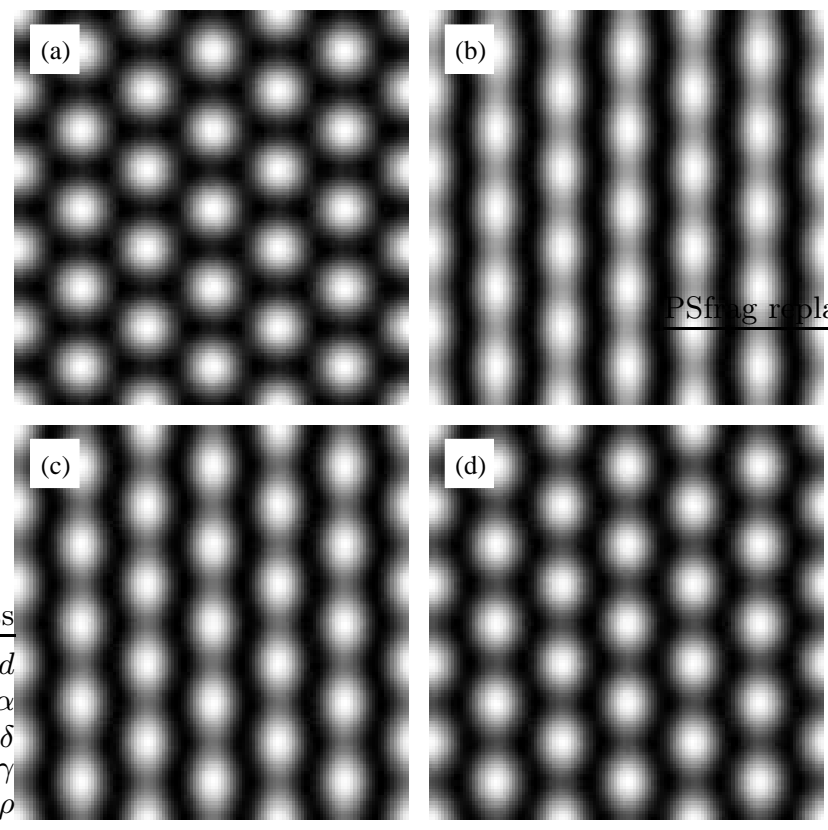

FIG. 9: The spatial variation of one term contributing to Eq. (10) is shown for hexagons in part (a) and for distorted hexagons in (b), (c) and (d). In part (b) a distorted hexagon is depicted for the parameters used in Fig. [6 (b), namely $\bar{G}_{1}=$ 0.05 and $\eta=-0.0492$ with the amplitudes being $A=0.0551$ and $B=0.0124$. In the remaining two parts the modulation amplitude is $\bar{G}_{1}=0.11$, whereas the control parameter is $\eta=$ -0.099 in (c) (with $A=0.15$ and $B=0.06$ ) and $\eta=-0.09$ in part (d) (with $A=0.198$ and $B=0.116$ ).

\section{Solutions of the forced amplitude equations for $k_{m}=k_{c}$ and $G_{1}=0, G_{2} \neq 0$.}

As in the previous case two amplitudes of the three mode solution coincide, i.e. $A_{2,3}=B e^{i \theta_{2,3}}$, whereas the third one is given by $A_{1}=A e^{i \theta_{1}}$. The two remaining moduli $A$ and $B$ are determined by the following nonlinear equations

$$
\begin{aligned}
& \left(\eta-|\bar{A}|^{2}-2 \bar{\rho}|\bar{B}|^{2}\right) \bar{A}+\bar{G}_{2}+e^{i \Theta} \bar{B}^{* 2}=0, \\
& \left(\eta-(1+\bar{\rho})|\bar{B}|^{2}-\bar{\rho}|\bar{A}|^{2}\right) \bar{B}+e^{i \Theta} \bar{A}^{*} \bar{B}^{*}=0 .
\end{aligned}
$$

If $B=0$ and $\bar{G}_{2} \neq 0$ the single mode solution is determined by a third order polynomial in $A$. In contrast to the case discussed in Sec. $\nabla B$ the bifurcation is now an imperfect one as can be seen in Fig. 10] Despite this imperfect bifurcation one may observe the following similarities to the previous paragraph wherein the $2: 1$ resonance was studied: First of all the degeneracy of three mode solutions encountered for $\bar{G}_{2}=0$ vanishes once again for finite values of the modulation amplitude $\bar{G}_{2}$. Secondly, as for the $2: 1$ resonance, $A$ corresponding to the largest amplitude of the three mode solution bifurcates for small values of $\bar{G}_{2}$ from the top of the single mode solution, whereas the small amplitude $B$ bifurcates from zero.
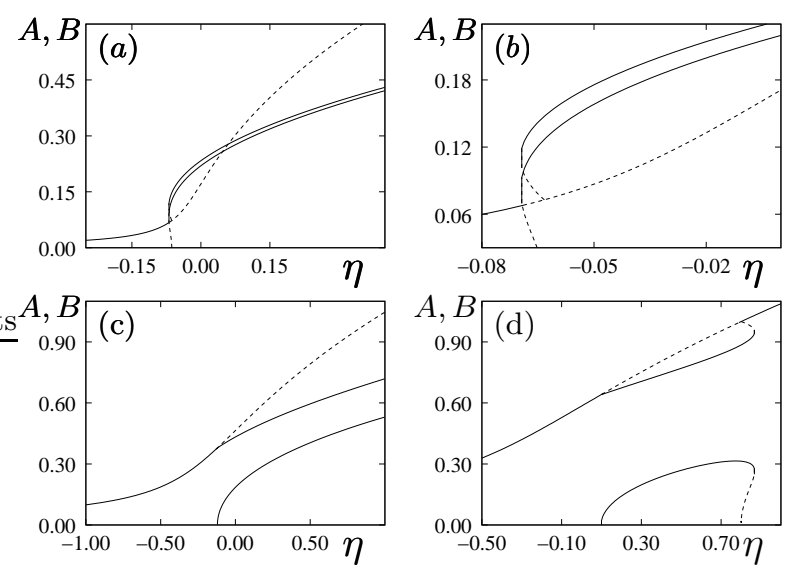

FIG. 10: The amplitudes of a three mode solution with $A$ always corresponding to the upper branch and $B$ to the lower one, as well as the amplitude of the imperfect bifurcating single mode solution $(B=0)$ are plotted as a function of the control parameter $\eta$. Three different values of the modulation amplitude are taken into account: $\bar{G}_{2}=0.05$ (a), where (b) is an enlargement of (a) over a smaller $\eta$-range, $\bar{G}_{2}=0.1$ (c) and $\bar{G}_{2}=0.2(\mathrm{~d})$. Solid lines mark linear stable solutions and dashed lines unstable ones. Furthermore $\bar{\rho}=1.7$ and $\bar{\delta}=1$.

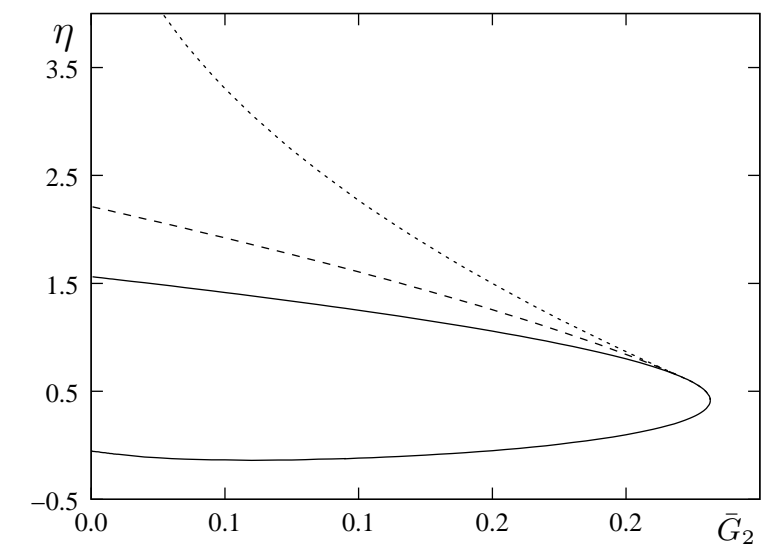

FIG. 11: In between the dotted line and the lower solid line (coinciding in the lower half-plane with the dashed line) three mode solutions exist. Along the dashed line, which overlaps on its lower branch with lower branch of the solid line, the values of the functionals of single and three mode solutions match. Thus within the area bounded by the dashed line three mode solutions are favored. Single mode solutions are linear stable in the complementary part of the region enclosed by the solid line. The nonlinear coefficient is assumed to be $\bar{\rho}=1.7$ and $\bar{\delta}=1$.

With increasing values of $\bar{G}_{2}$ the bifurcation of the three mode solution changes once more at its lower bifurcation point from a subcritical one in Fig. 10(a) to a supercritical one in Fig. 10/c) or in Fig. 10(d). The bifurcation point located at large values of $\eta$ is not shown in Fig. 10 (a) and Fig. 10(c) but is subcritical as the one depicted in Fig. 7 for a $2: 1$ resonance. Thirdly the hysteresis described by the three mode solution becomes 
once again less pronounced with increasing values of $\bar{G}_{2}$ and turns out to be supercritical for large modulation amplitudes. Last but not least the three mode solution ceases to exist beyond some critical value of the modulation amplitude $\bar{G}_{2}$ as it was noticed as well for a $2: 1$ resonance.

The properties explained in the previous paragraph are recapitulated in Fig. [1] where three mode solutions exist within the region bounded by the dotted lines, overlapping in the lower half-plane with the solid line. Along the dashed line the functionals $\mathcal{F}$ of the single and three mode solution match, meaning that the three mode solution is preferred in between the dashed lines coinciding once more with the solid line for small values of the control parameter $\eta$. This overlapping of lines is due to the fact that the lower bifurcation point is supercritical for small $\bar{G}_{2}$ and that the extension of the hysteresis shrinks with increasing modulation amplitudes. Through a linear stability analysis, similar to the one described in Sec. IVC one may conclude that single mode solutions are linear stable only outside of the area delimited by the solid line.

\section{NUMERICAL RESULTS}

A major problem always encountered when dealing with amplitude equations is that their validity range around the threshold remains a priori unpredictable. Therefore we have determined the amplitude of the stripe solution by numerical simulations of the basic Eqs. (11) without considering modulations as a function of the relative distance $\eta$ from threshold and compared these numerical results with the analytical solution $A=\sqrt{\eta / \gamma}$ in Fig. 12. Up to $\eta \sim 0.2$ we find a fairly good agreement between analytical and numerical results, which gives a reasonable estimate of the quantitative validity range of the preceding results obtained in terms of amplitude equations.

\section{SUMMARY AND CONCLUSIONS}

The generic amplitude equations (13) are suggested for a forced two-dimensional system with a broken up-down symmetry and discussed throughout this work. Additionally they are derived for the first time explicitly from a model for chemical reactions, in the present case the Lengyel-Epstein model. They are apt to describe how spatial periodic modulations interfere with the competition between stripes and hexagonal patterns. Our interest was particularly focused on a $1: 1$ and a $2: 1$ resonance between the modulation wave number $k_{m}$ and the wave number $k_{c}$ of the respective pattern. Compared to the unmodulated case the bifurcation-scenarios of stripes and hexagonal patterns change strongly in the presence of modulations. It has been shown that three mode solutions, i.e. hexagons in the unmodulated case and dis-

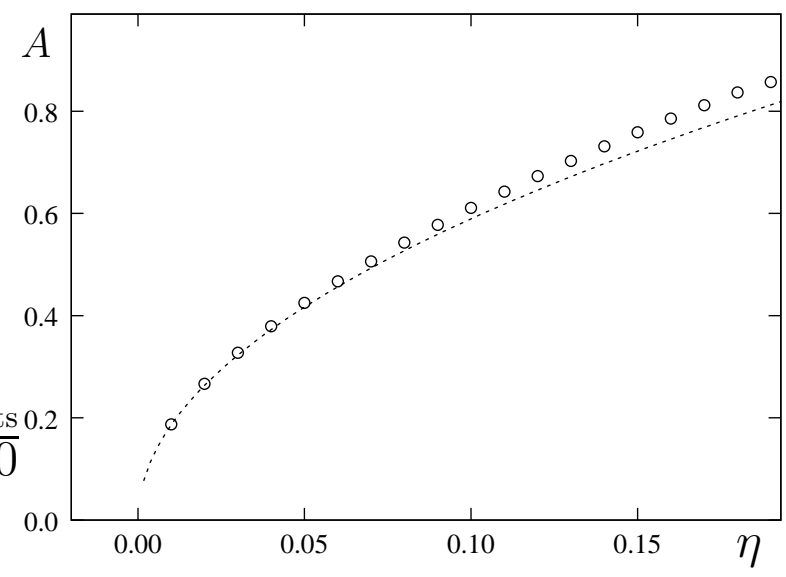

FIG. 12: The amplitude of a stripe solution obtained by numerical simulations (circles) of the microscopic model given by Eqs. (1) is compared with the analytical solution $A=\sqrt{\eta / \gamma}$. The parameters used are $a=16, \sigma=301, d=1.07, c=0.6$ which yield the nonlinear coefficient $\gamma=0.288$. For the sake of simplicity no modulations were taken into account.

torted hexagons otherwise, are suppressed for large modulation amplitudes. Furthermore evidence was given that depending on the strength of the modulation amplitude those three mode solutions bifurcate either sub- or supercritically. The bifucation diagrams presented in this work may be confirmed by experiments on chemical reactions with a spatially modulated illumination, where in contrast to Refs. [20, 21] the ratio between the wave number of the illumination and the wave number of the Turing pattern should be chosen to be $2: 1$ or $1: 1$.

Instead of considering a stationary forcing the effect of traveling stripe forcing on the competition between hexagons and stripes has recently been investigated for a $1: 1$ resonance in a one-dimensional system [34]. Given the coupled amplitude equations (13) the results established in Ref. 34 may be directly interpreted within the scope of the microscopic Lengyel-Epstein model. The same allegation holds for the $1: 1$ resonance analyzed in a two-dimensional system in Ref. [35]. Although the bifurcation-scenarios of the stationary $1: 1$ and $2: 1$ resonance we studied in the present work share some similarities, there remains the open question how the bifurcation diagrams presented in Refs. [34, 35] are modified if one has to deal with a $2: 1$ resonance instead of a $1: 1$ resonance.

We mainly focused on the spatially periodic forcing of Turing patterns. The Lengyel-Epstein model exhibits furthermore a Hopf-bifurcation [56] similar to those found in other chemical reaction schemes. Instead of a temporal resonant forcing of an oscillatory but spatially homogeneous chemical reaction [31, 32 one may induce such an oscillatory reaction by a spatially periodic or a traveling stripe modulation. This leads to a revealing amplitude equation, which has been recently deduced from the Lengyel-Epstein model and exhibits rich transition scenarios between solutions that are either spatially har- 
monic or subharmonic with respect to the external modulation [57].
This work results mainly from a summer project for students.
[1] R. E. Kelly and D. Pal, J. Fluid Mech. 86, 433 (1978).

[2] M. Lowe, J. P. Gollub, and T. Lubensky, Phys. Rev. Lett. 51, 786 (1983).

[3] M. Lowe, B. S. Albert, and J. P. Gollub, J. Fluid Mech. 173, 253 (1986).

[4] P. Coullet, Phys. Rev. Lett. 56, 724 (1986).

[5] P. Coullet and P. Huerre, Physica (Nonlin. Phenomena) D 23, 27 (1986).

[6] D. A. S. Rees and D. S. Riley, J. Fluid Mech. 166, 503 (1986).

[7] P. Coullet and D. Walgraef, Europhys. Lett. 10, 525 (1989).

[8] G. Hartung, F. H. Busse, and I. Rehberg, Phys. Rev. Lett. 66, 2741 (1991).

[9] W. Zimmermann, in Defects, Singularities in Nematic Liquid Crystals: Mathematical and Physical Aspects, Vol. 332 of NATO ASI Series C, edited by J. M. Coron, J. M. Ghidaglia, and F. Helein (Kluwer, Dordrecht, 1991), p. 401.

[10] W. Zimmermann et al., Europhys. Lett. 24, 217 (1993).

[11] W. Zimmermann and R. Schmitz, in Spatio-Temporal Patterns in Nonequilibrium Complex Systems, edited by P. Cladis and P. Palffy-Muhoray (Addison-Wesley, New York, 1994), p. 273.

[12] A. Ogawa, W. Zimmermann, K. Kawasaki, and T. Kawakatsu, J. Phys. II (Paris) 6, 305 (1996).

[13] W. Zimmermann and R. Schmitz, Phys. Rev. E 53, R1321 (1996).

[14] R. Schmitz and W. Zimmermann, Phys. Rev. E 53, 5993 (1996).

[15] R. Schmitz and W. Zimmermann, Phys. Lett. A 237, 405 (1997).

[16] A. D. Stroock, R. F. Ismagilov, H. A. Stone, and G. M. Whitesides, Langmuir 19, 4358 (2003).

[17] W. Zimmermann, B. Painter, and R. Behringer, Eur. Phys. J. B 5, 575 (1998).

[18] Y. Hidaka, T. Fujimura, N. A. Mori, and S. Kai, Mol Cryst. \& Liq. Cryst. 328, 1999 (1999).

[19] R. Neubecker and A. Zimmermann, Phys. Rev. E 65, 035205 (2002).

[20] M. Dolnik, I. Berenstein, A. M. Zhabotinsky, and I. R. Epstein, Phys. Rev. Lett. 87, 238301 (2001).

[21] I. Berenstein et al., Phys. Rev. Lett. 91, 058302 (2003).

[22] M. Henriot, J. Burguette, and R. Ribotta, Phys. Rev. Lett. 91, 104501 (2003).

[23] G. Ahlers, P. C. Hohenberg, and M. Lücke, Phys. Rev. Lett. 53, 48 (1984).

[24] G. Ahlers, P. C. Hohenberg, and M. Lücke, Phys. Rev. A 32, 3493 (1985).

[25] H. Riecke, J. D. Crawford, and E. Knobloch, Phys. Rev. Lett. 61, 1942 (1988).

[26] D. Walgraef, Europhys. Lett. 7, 485 (1988).

[27] I. Rehberg et al., Phys. Rev. Lett. 61, 2449 (1988).

[28] P. Coullet and K. Emillson, Physica (Nonlin. Phenomena) D 61, 119 (1992).
[29] M. Silber, H. Riecke, and L. Kramer, Physica (Nonlin. Phenomena) D 61, 260 (1992).

[30] J. L. Rogers, M. L. Schatz, O. Brausch, and W. Pesch, Phys. Rev. Lett. 85, 4281 (2000).

[31] A. L. Lin et al., Phys. Rev. Lett. 84, 4240 (2000).

[32] A. L. Lin et al., Phys. Rev. E 62, 3790 (2000).

[33] C. Utzny, W. Zimmermann, and M. Bär, Europhys. Lett. 57, 113 (2002).

[34] S. Rüdiger et al., Phys. Rev. Lett. 90, 128301 (2003).

[35] D. G. Miguez et al., Phys. Rev. Lett. 93, 048303 (2004).

[36] S. Schuler, M. Hammele, and W. Zimmermann, Traveling stripe forcing of oblique rolls in anisotropic systems, accepted for publ. in European Phys. J B, 2004.

[37] F. H. Busse, J. Fluid Mech. 30, 625 (1967).

[38] F. H. Busse, in Convection: Plate Tectonics and Global Dynamics, edited by W. R. Peltier (Gordon and Breach, Montreux, 1989), pp. 23-95.

[39] A. Turing, Phil. Trans. R. Soc. London B 237, 37 (1952).

[40] J. D. Murray, Mathematical Biology (Springer, Berlin, 1989).

[41] P. G. de Gennes and J. Prost, The Physics of Liquid Crystals (Clarendon, Oxford, 1993).

[42] S. Kai and W. Zimmermann, Prog. Theor. Phys. Suppl. 99, 458 (1989).

[43] L. Kramer and W. Pesch, Annu. Rev. Fluid Mech. 27, 515 (1995).

[44] A. Buka and L. Kramer, Pattern Formation in Liquid Crystals (Springer, Berlin, 1996).

[45] R. Schmitz and W. Zimmermann, J. Phys. II France 7, 677 (1997).

[46] R. Friedrichs, Phys. Rev. E 66, 066215 (2002).

[47] T. Ackemann, B. Giese, B. Schapers, and W. Lange, J. Optics B 1, 70 (1999).

[48] V. K. Vanag and I. R. Epstein, Phys. Rev. E 67, 066219 (2003).

[49] I. Lengyel and I. R. Epstein, Science 251, 650 (1990).

[50] A. P. Munuzuri, M. Dolnik, A. M. Zhabotinsky, and I. R. Epstein, J. Am. Chem. Soc. 121, 8065 (1999).

[51] A. Sanz-Anchelergues, A. M. Zhabotinsky, I. R. Epstein, and A. P. Munuzuri, Phys. Rev. E 63, 056124 (2001).

[52] M. C. Cross and P. C. Hohenberg, Rev. Mod. Phys. 65, 851 (1993).

[53] P. Manneville, Dissipative Structures and Weak Turbulence (Academic Press, London, 1990).

[54] S. Ciliberto et al., Phys. Rev. Lett. 65, 2370 (1990).

[55] M. Hilt and W. Zimmermann, On hexagonal, square and stripe pattern of the ion-channel density in biomembranes, preprint, 2004.

[56] I. Lengyel, S. Kádár, and I. R. Epstein, Science 259, 493 (1993).

[57] M. Hammele and W. Zimmermann, Effects of spatial periodic modulation on an oscillating chemical reaction, preprint, 2004. 